\title{
e-Phaïstos
}

e-Phaïstos

Revue d'histoire des techniques / Journal of the history

of technology

II-2 | 2013

Les sources de l'histoire des techniques (2)

\section{La tenue du violon à l'époque baroque}

Techniques for Holding the Violin during the Baroque Period

\section{Cyril Lacheze}

\section{(2) OpenEdition}

Journals

Édition électronique

URL : https://journals.openedition.org/ephaistos/9245

DOI : 10.4000/ephaistos.9245

ISSN : 2552-0741

Éditeur

IHMC - Institut d'histoire moderne et contemporaine (UMR 8066)

Édition imprimée

Date de publication : 15 décembre 2013

Pagination : 28-42

ISSN : 2262-7340

Référence électronique

Cyril Lacheze, "La tenue du violon à l'époque baroque », e-Phaïstos [En ligne], II-2 | 2013, mis en ligne le 15 décembre 2020, consulté le 16 septembre 2021. URL : http://journals.openedition.org/ephaistos/ 9245 ; DOI : https://doi.org/10.4000/ephaistos.9245

Ce document a été généré automatiquement le 16 septembre 2021.

Tous droits réservés 


\title{
La tenue du violon à l'époque baroque
}

\author{
Techniques for Holding the Violin during the Baroque Period
}

\author{
Cyril Lacheze
}

1 Les études concernant les arts et plus particulièrement la musique restent encore peu développées en histoire des techniques, bien que quelques publications à ce sujet aient déjà été écrites, notamment à travers la question de la réduction en $\operatorname{art}^{1}$. Pourtant, la musique semble un objet d'étude intéressant, dans le sens où avant d'être un art, elle est avant tout technique. En effet, seule l'interprétation relève véritablement d'une démarche artistique ; la théorie musicale, elle, est fondamentalement mathématique, et c'est l'exécution qui requiert un très haut niveau de technicité. C'est sur ce dernier aspect, souvent largement ignoré par les études purement musicales (on préfère s'intéresser aux compositeurs ou à leurs œuvres), que nous nous proposons de nous pencher, à travers la question de la tenue du violon à l'époque baroque.

2 Ce sujet, que nous traitons plus amplement à travers un mémoire de Master, peut a priori paraitre bien anecdotique. Il s'insère pourtant dans un mouvement de fond développé dans le monde de la musique depuis les années 1970, celui de la musique ancienne. Son principe est assez proche de celui de l'« histoire appliquée » : il s'agit de jouer les œuvres non pas avec les goûts, habitudes et techniques actuels, pour la plupart hérités directement de l'école romantique du XIX ${ }^{e}$ siècle, mais de retrouver les manières, sonorités et instruments contemporains de chaque pièce. La période d'exercice privilégiée de cette méthodologie a été dès le départ la période moderne, correspondant dans le domaine musical à la Renaissance (1500-1580), au baroque ancien (1580-1630), moyen (1630-1700) et récent (1700-1750), et à la période classique (1750-1800). Ce mouvement s'étend actuellement tout aussi bien au Moyen Age et au XIX ${ }^{e}$ siècle romantique, mais nous nous focaliserons pour cette étude sur la période moderne.

3 En effet, celle-ci correspond à la période d'apparition et de stabilisation à la fois du violon et de sa technique de jeu. Documenté pour la première fois dans la décennie 1530, en Italie du Nord pour l'iconographie et en Savoie pour les sources textuelles, cet 
instrument dérive des vièles et violes de la fin du Moyen Age, sans que son origine exacte puisse être retracée. D'abord peu estimé par rapport à la viole et plutôt destiné aux danses paysannes, le violon s'est rapidement imposé dans le répertoire musical au cours du XVII ${ }^{e}$ siècle, jusqu'à devenir incontournable à partir du XVIII ${ }^{\mathrm{e}}$ siècle. Parallèlement à ce développement dans le répertoire, ses techniques ont évolué vers une virtuosité plus importante, au détriment d'un jeu plus instinctif prévalant au début de son existence. Dans le même temps, la tenue à la fois du violon et de l'archet ont également beaucoup varié, selon des modalités encore mal comprises, pour se stabiliser seulement à partir du classicisme et surtout du romantisme (soit au tournant des XVIII ${ }^{\mathrm{e}}$ et XIX ${ }^{e}$ siècles) dans la forme connue actuellement. Or, si l'évolution finale vers la tenue actuelle est bien connue et étudiée, les multiples modifications qui sont intervenues auparavant, entre le $\mathrm{XVI}^{\mathrm{e}}$ et le milieu du XVIII ${ }^{\mathrm{e}}$ siècle, restent en grande partie inconnues, ce à quoi nous allons tenter de remédier dans cette étude.

\section{Une recherche novatrice}

\section{Historiographie}

4 De fait, l'historiographie sur cette question est extrêmement mince. Aucune étude ancienne n'existe, simplement parce que cette question n'était pas posée jusqu'à assez récemment. Si certains professionnels et amateurs du XIX ${ }^{e}$ siècle ont commencé à redécouvrir les compositeurs de la première moitié du XVIII ${ }^{\mathrm{e}}$ siècle, l'idée d'une interprétation historiquement réfléchie leur était totalement étrangère (l'interprétation "académique » actuelle de ces musiques provenant directement de cette conception). De même, au début du $\mathrm{XX}^{\mathrm{e}}$ siècle, quelques essais ont été tentés pour faire revivre les instruments d'époque (notamment le clavecin sous l'impulsion de Maria Landowska), mais la technique n'était aucunement prise en considération. Ainsi, les quelques études historiques sur le violon entre le $\mathrm{XIX}^{\mathrm{e}}$ siècle et le milieu du $\mathrm{XX}^{\mathrm{e}}$ siècle n'ont jamais abordé cette problématique.

Le principal jalon à noter ensuite est la parution de The History of Violin Playing from Its Origins to $1761 \mathrm{du}$ musicologue et violoniste David Boyden, en $1965^{2}$ : si le propos est assez généraliste, la taille imposante de cet ouvrage a permis à l'auteur de consacrer plusieurs pages à la question de la tenue de l'instrument, sur une période chronologique résolument ancienne. Il s'agit toujours de la référence sur ces questions à l'heure actuelle, mais il faut noter que ce livre est paru quelques années avant le début de la pratique « historiquement informée » de la musique ancienne : il n'intègre donc pas les observations réalisées à partir de celle-ci, et, la réédition de 1990 n'ayant quasiment rien changé de l'original, il est aujourd'hui dépassé.

6 Très peu d'auteurs se sont penchés sur cette question depuis les travaux de David Boyden, hormis dans quelques publications reprenant exactement les mêmes analyses. La seule étude se rapportant spécifiquement à la tenue du violon est un petit article du violoniste Jean-Paul Burgos, paru en 1985 dans une revue consacrée à la flûte à bec et passé semble-t-il totalement inaperçu ${ }^{3}$. Il faut pour finir citer l'excellent ouvrage de la musicologue Greta Moens-Haenen sur le violon en Allemagne au XVIIe siècle, qui consacre une quarantaine de pages à la question de la tenue mais uniquement dans les limites chronologique et géographique indiquées ${ }^{4}$. A quelques très rares exceptions près, traitant notre sujet de manière périphérique ${ }^{5}$, l'historiographie existante est donc 
constituée de seulement trois ouvrages, dont le seul traitant en profondeur de l'intégralité de la question date d'il y a presque un demi-siècle.

7 De plus, la pratique actuelle du violon baroque est en grande partie liée à la formation dispensée en conservatoire. Or, celle-ci s'est rapidement "standardisée » sur une configuration particulièrement simple à apprendre pour des étudiants ayant quasiment tous reçu auparavant une formation de violon moderne, à savoir la tenue la plus moderne du violon baroque, en vigueur à partir de la deuxième moitié du XVIIIe siècle. Ainsi, outre le fait que les universitaires ne se soient quasiment pas penchés sur la question, les musiciens eux-mêmes ne s'y intéressent pas vraiment non plus, et les quelques-uns à l'avoir fait (Amandine Beyer ou Odile Edouard par exemple), n'ont pas poussé la réflexion jusqu'à aborder des tenues très éloignées de la pratique habituelle. Notre recherche se situe donc dans un champ très peu abordé à l'heure actuelle, par quelque discipline que ce soit, et qui pourtant trouverait tout à fait sa place dans les dynamiques de recherches à l'œuvre depuis quelques décennies ${ }^{6}$.

\section{Sources disponibles}

8 Les sources disponibles pour une telle étude sont variées et relativement nombreuses, mais de nature parfois différente des sources habituelles de l'histoire des techniques. Les écrits [fig.1] existent bien, sous forme de traités, mais ceux-ci sont peu nombreux (une petite trentaine), inégalement répartis et de qualité très variable (même les plus importants ne comportent que quelques lignes utiles). Les traités du XVI ${ }^{e}$ et du début $\mathrm{du} \mathrm{XVII}$ e siècles sont presque inexistants : en effet, de nombreux ouvrages de réduction en art existent, notamment en France, mais l'on réduit en art la musique et non le violon. Dans ce sens, les instruments ne sont considérés que comme une mise en pratique d'une conception totalement théorique de la musique, et ne sont donc généralement pas étudiés, et s'ils le sont, la tenue n'est pour ainsi dire jamais évoquée. Quelle que soit l'époque, il n'est ainsi jamais fait mention de la tenue dans les traités italiens. La seconde moitié du XVII ${ }^{\mathrm{e}}$ siècle est dominée largement par les écrits allemands, mais, à une exception près, ceux-ci sont en réalité plutôt à considérer comme des manuels pour débutants : ils contiennent surtout des exercices, à réaliser explicitement sous la houlette d'un professeur, avec simplement quelques pages d'introduction. 
Figure 1. Répartition spatio-temporelle des sources écrites

\begin{tabular}{|c|c|c|c|c|c|}
\hline & & Espagne & France & Allemagne & Angleterre \\
\hline \multirow{2}{*}{ XVIe siècle } & $3^{e}$ quart & & 1 & & \\
\hline & $4^{\mathrm{e}}$ quart & & 1 & & \\
\hline \multirow{4}{*}{ XVII' siècle } & $1^{\mathrm{er}}$ quart & & & 1 & \\
\hline & $2^{e}$ quart & & 1 & & \\
\hline & $3^{\mathrm{e}}$ quart & & & & 1 \\
\hline & $4^{e}$ quart & & & 5 & 1 \\
\hline \multirow{4}{*}{$\begin{array}{l}\text { XVIIIe } \\
\text { siècle }\end{array}$} & $1^{\text {er }}$ quart & & 2 & & 1 \\
\hline & $2^{\mathrm{e}}$ quart & & 1 & 1 & \\
\hline & $3^{\text {e }}$ quart & 1 & 4 & 4 & 2 \\
\hline & $4^{\mathrm{e}}$ quart & & 2 & & \\
\hline
\end{tabular}

Tableau C. Lachèze

Il faut donc attendre le XVIII ${ }^{\mathrm{e}}$ siècle, avec encore uniquement des écrits allemands dans le premier tiers puis la réapparition d'écrits français ensuite, pour voir apparaitre de vrais traités, comportant encore une grande majorité d'exercices, mais assortis d'une réflexion plus poussée sur les principes de jeu. Cette amélioration des informations à disposition, qui explique l'attachement quasi exclusif des musiciens à la technique de cette période, culmine avec les traités des violonistes et compositeurs Francesco Geminiani $^{7}$ (1751) et Leopold Mozart ${ }^{8}$ (1770), très tardifs pour notre étude mais précurseurs des réels ouvrages méthodiques du XIX siècle. Il faut enfin noter l'existence d'une lignée de traités anglais dans la seconde moitié du XVII et première moitié du XVIII ${ }^{e}$ siècles, tous très semblables, se composant presque exclusivement de mélodies simples à destination d'amateurs et n'apportant donc que des informations limitées ou très spécifiques.

Parallèlement à ces sources textuelles, existe une très importante documentation iconographique, qui n'a malheureusement quasiment pas été exploitée jusqu'à présent. Pourtant, nous avons pu recenser environ 600 attestations de violons ou instruments assimilés entre le Moyen Age et la fin du XVIII ${ }^{e}$ siècle (ce qui n'est certainement pas exhaustif), dont plus de la moitié en position de jeu [fig.2]. La musique en elle-même constitue également une source, à travers les partitions. En effet, il est parfois possible de supposer, voire d'affirmer, une corrélation entre une technique et un résultat sonore, l'analyse de certains morceaux pouvant donc nous renseigner sur la tenue employée par leur compositeur ou ses contemporains. Enfin, il nous reste à évoquer quelques parallèles possibles du point de vue ethnographique, avec la musique populaire encore pratiquée dans certains pays (Maghreb, Scandinavie par exemple). Mais c'est surtout l'expérimentation, qui tient une place toute particulière dans cette étude. En effet, elle permet de tester concrètement les hypothèses formulées et de détecter certaines incohérences physiques; mais aussi, en tant que telle, de formuler certaines hypothèses. Enfin, au contraire de nombreux domaines de recherche, la finalité première de la musique restant la représentation en public, dans le cas de la musique ancienne, une "reconstitution " peut donc faire office de médiation, sur les modalités de laquelle il convient impérativement de mener une réflexion approfondie. 
Figure 2. Répartition spatio-temporelle des sources iconographiques

\begin{tabular}{|c|c|c|c|c|c|c|}
\hline & $\begin{array}{c}\text { Italie } \\
\text { Espagne }\end{array}$ & $\begin{array}{c}\text { France } \\
\text { Suisse }\end{array}$ & $\begin{array}{c}\text { Allemagne } \\
\text { Bohême }\end{array}$ & $\begin{array}{c}\text { Hollande } \\
\text { Belgique }\end{array}$ & Angleterre & Total \\
\hline Moyen-Age (avant 1500) & 57 & 5 & 13 & 3 & 1 & 79 \\
\hline Renaissance (1500-1580) & 40 & 1 & 17 & 9 & 1 & 68 \\
\hline Baroque ancien (1580-1630) & 24 & 5 & 1 & 44 & 4 & 78 \\
\hline Baroque moyen (1630-1700) & 5 & 4 & 1 & 44 & 0 & 54 \\
\hline Baroque récent (1700-1750) & 10 & 13 & 7 & 0 & 11 & 41 \\
\hline Classicisme (1750-1800) & 2 & 5 & 5 & 0 & 2 & 14 \\
\hline Total & 138 & 33 & 44 & 100 & 19 & 334 \\
\hline
\end{tabular}

Tableau C. Lachèze

\section{Premiers résultats}

\section{Typo-chronologie}

La bibliographie existante, lorsqu'elle synthétise les quelques recherches spécifiques à ce sujet, se contente d'une typo-chronologie très sommaire quant aux différentes tenues de l'époque baroque. Le violon se serait tenu au niveau du cou à la Renaissance, héritage des vièles médiévales, avant de redescendre vers l'épaule ou la poitrine au $\mathrm{XVII}^{\mathrm{e}}$ siècle, puis de remonter pour se stabiliser de nouveau à hauteur du cou au XVIII ${ }^{\mathrm{e}}$ siècle, plus ou moins comme actuellement. Quant à l'archet, on distingue, en suivant en cela une ou deux sources assez tardives ${ }^{9}$, une tenue italienne, avec la main très avancée sur l'archet, et une tenue française plus proche de la technique actuelle à quelques détails près [fig.3]. Une analyse approfondie des sources disponibles montre cependant que, si elle n'est pas fondamentalement fausse, une telle description est extrêmement réductrice et nécessite de très nombreuses nuances [fig.4].

Figure 3. Tenues d'archet d'après l'analyse de Michel Corrette (1738) 


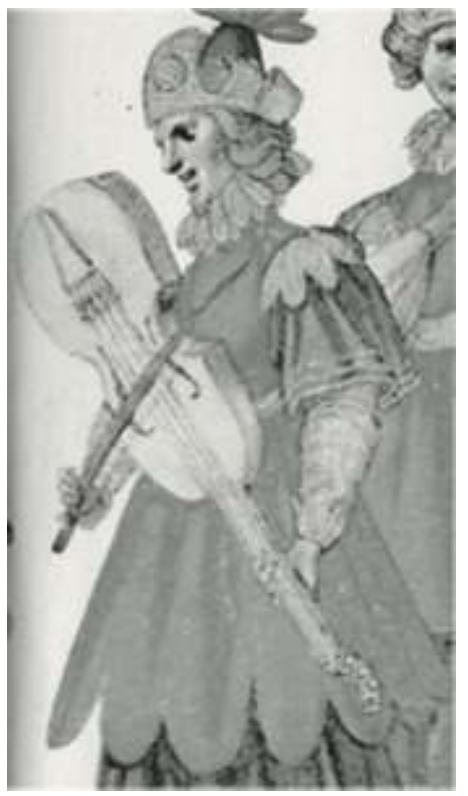

Tenue « française » (à l'extrémité de l'archet, le pouce sous la hausse). L'instrument représenté n'est pas un violon mais probablement un violoncello da spalla.

RABEL Daniel, Ballet des fées de la forêt de Saint Germain, 1625, p.15. Détail.

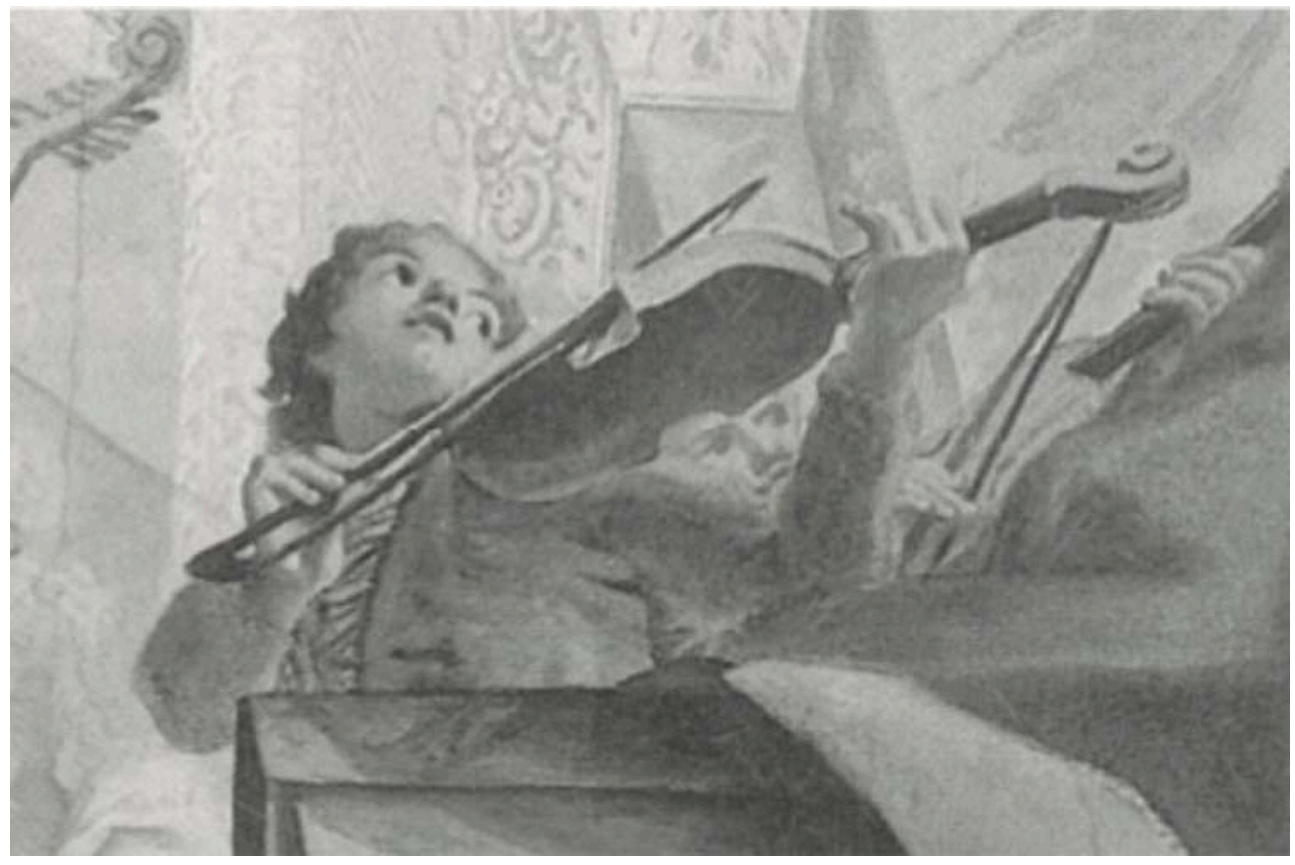

Tenue « italienne» (la main avancée sur la baguette).

ASAM, Cosmas, Musiciens à la fête de la Saint Günther, 1726-1728, fresque, Brevnov, Prälatensaal im ehemaligen Benediktinerkloster. Détail. 
Figure 4. Les grandes tendances techniques d'après les sources iconographiques hors contexte religieux, le Moyen Age et l'époque classique ne nous fournissant que très peu d'informations.

\begin{tabular}{|c|c|c|c|c|c|c|c|c|c|c|}
\hline & \multicolumn{2}{|c|}{$\begin{array}{c}\text { Italie } \\
\text { Espagne }\end{array}$} & \multicolumn{2}{|c|}{$\begin{array}{l}\text { France } \\
\text { Suisse }\end{array}$} & \multicolumn{2}{|c|}{$\begin{array}{l}\text { Allemagne } \\
\text { Bohême }\end{array}$} & \multicolumn{2}{|c|}{$\begin{array}{l}\text { Hollande } \\
\text { Belgique }\end{array}$} & \multicolumn{2}{|c|}{ Angleterre } \\
\hline & v1. & Ar. & VI. & Ar. & Vl. & Ar. & Vl. & Ar. & v1. & Ar. \\
\hline Renaissance (1500-1580) & \multirow{2}{*}{ Basse } & \multirow{2}{*}{ Fr. } & & & & & & & & \\
\hline Baroque ancien (1580-1630) & & & Basse & \multirow{3}{*}{ Fr. } & & & \multirow{2}{*}{ Basse } & Fr. & & \\
\hline Baroque moyen (1630-1700) & & & & & & & & Var. & Var. & \multirow{2}{*}{ It. } \\
\hline Baroque récent (1700-1750) & Haute & Var. & & & Haute & It. & & & Haute & \\
\hline
\end{tabular}

Abréviations : VI. (Violon), Ar. (Archet), Fr. (Française), It. (Italienne), Var. (Variable).

Tableau C. Lachèze

Figure 5. Quelques exemples de tenues courantes

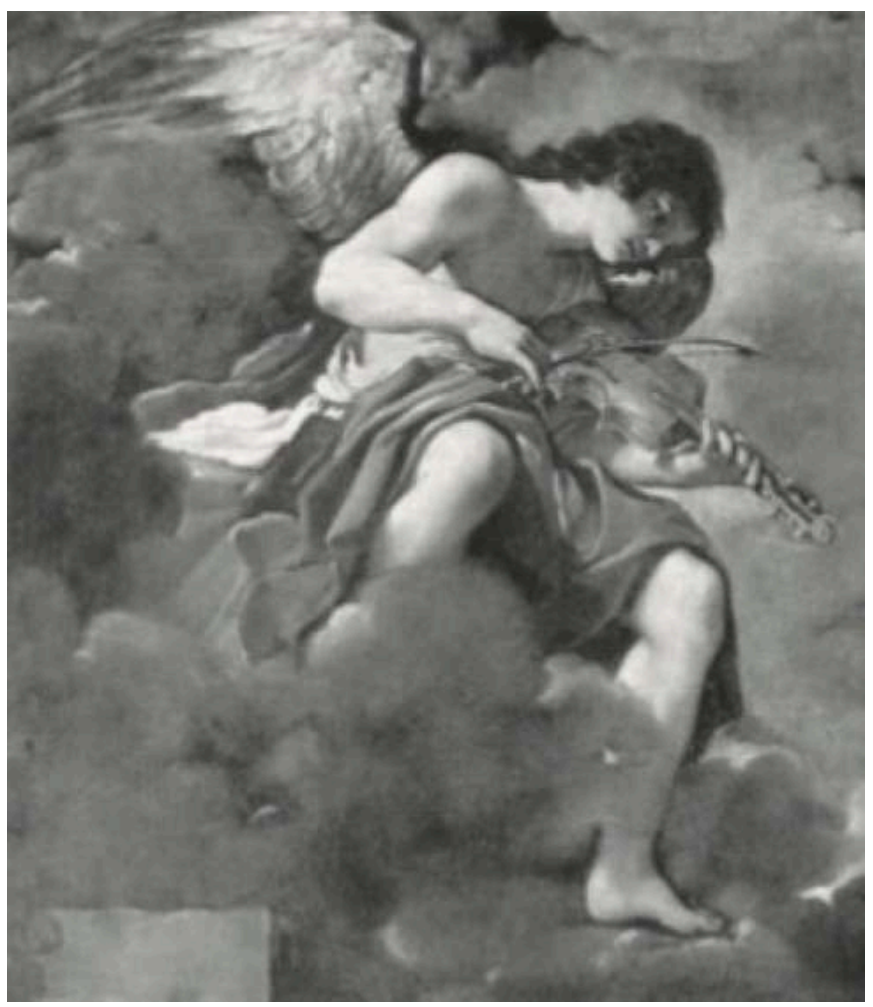

Violon bas, archet tenu à la française

GUERCINO, Saint François avec un ange jouant du violon, 1620, huile sur toile, $162 \times 127 \mathrm{~cm}$, Dresde, Gemäldegalerie. Détail. 


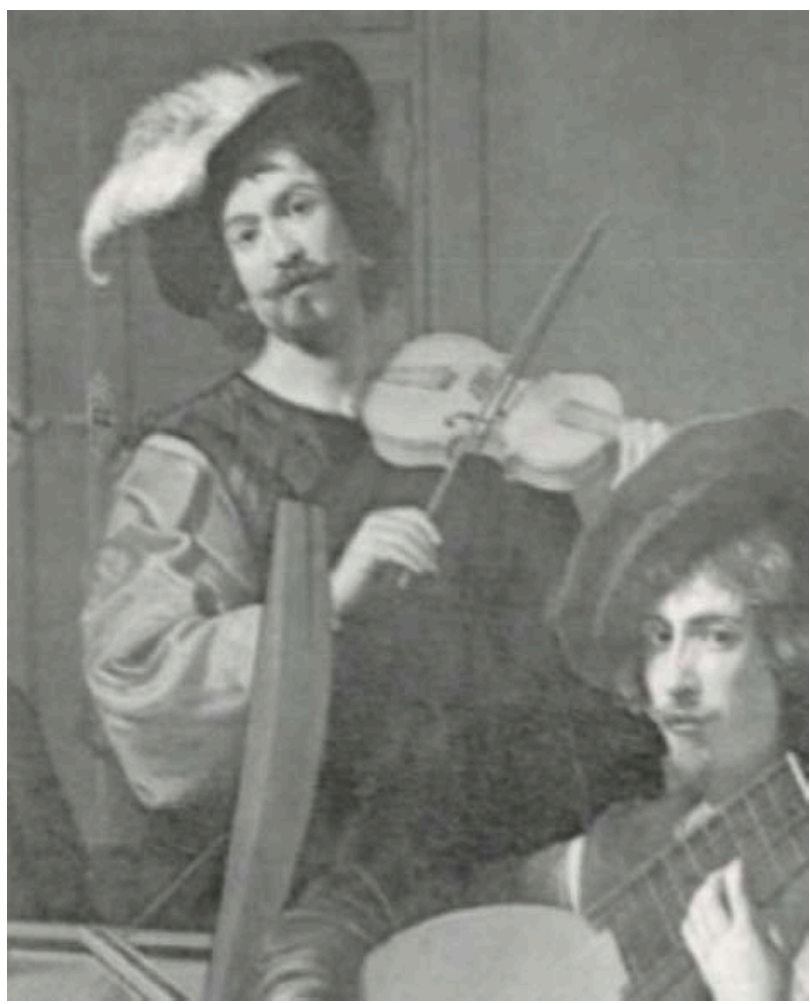

Violon haut, archet tenu à la française.

TOURNIERES, Robert, Concert, v.1690-1700, huile sur toile, NC, Paris, Musée du Louvre. Détail.

En premier lieu, au travers à la fois des sources écrites et iconographiques, nous avons pu distinguer nettement plus de variantes de tenues que celles décrites jusque-là. En effet, pour ce qui est de la position du violon, seules les deux ou trois plus répandues étaient documentées, alors que nous pouvons en réalité en identifier près d'une dizaine, sans prendre en compte les différentes variantes de chacune : on compte une demi-douzaine de positions différentes entre le cou et quasiment le niveau de la ceinture, en passant par l'épaule gauche, auxquelles il faut ajouter la possibilité de placer l'instrument contre l'épaule droite, sous le bras droit ou encore à la verticale. De même, les possibilités sont bien plus nombreuses pour l'archet qu'indiquées dans la bibliographie. Il est cependant bien évident que certaines de ces positions ne se rencontrent que de manière anecdotique : nous nous proposons donc de donner ici les grandes périodisations discernables avant de nous pencher sur quelques cas particuliers.

La tenue des instruments médiévaux se rapprochant du violon sont connues principalement par des peintures à thème religieux, dans les mains d'anges notamment, ce qui ne permet pas forcément de les considérer comme parfaitement fiables. Toutefois, elles montrent pour la plupart effectivement une tenue haute, entre le cou et l'épaule, qui reste d'ailleurs plus ou moins identique jusqu'au XVIII ${ }^{e}$ siècle : la représentation en contexte religieux du geste technique semble donc immuable, alors que celui-ci a en réalité évolué. Il est donc plus intéressant de se tourner vers les documents iconographiques représentant des scènes "réalistes", à la fois plus crédibles et comparables aux sources textuelles. Le XVI ${ }^{e}$ siècle n'est connu quasiment qu'à travers des tableaux italiens : ceux-ci montrent une tenue proche de celle des tableaux religieux, donc assez haute, ce qui correspond aux indications de la 
bibliographie. Comme indiqué également, le XVII ${ }^{e}$ siècle voit la position du violon s'abaisser vers la poitrine et l'épaule, mais il faut remarquer que les sources iconographiques sont alors françaises et hollandaises et non plus italiennes; en particulier, en Hollande, une légère distinction semble prendre place entre les pratiques populaire et bourgeoise, particulièrement à partir de la décennie 1630, la tenue la plus proche de la technique actuelle étant celle du bas peuple contrairement à ce que l'on pourrait penser. La situation semble globalement comparable en Allemagne d'après les sources textuelles, malgré les remises en cause de quelques théoriciens et notamment l'organiste Johann Prinner ${ }^{10}$.

Cependant, les situations se diversifient au XVIII e siècle : en effet, alors que les sources textuelles recommandent presque toutes de tenir l'instrument au niveau du cou, une tenue sur la poitrine reste admise très tardivement par certains auteurs, et notamment par Leopold Mozart en $1770^{11}$. De plus, dans l'iconographie, l'Allemagne et dans une moindre mesure l'Angleterre, semblent effectivement adopter une posture résolument moderne, alors que la France et l'Italie restent un peu plus traditionnelles. Surtout, pour l'archet, qui accepterait théoriquement deux tenues (française et italienne) se distinguant par la place de la main sur la baguette, on s'aperçoit facilement que si les français emploient bien la tenue qui porte leur nom, la situation est beaucoup moins évidente en Italie, et c'est en réalité l'Allemagne qui fait l'usage le plus exclusif de cette tenue «italienne ». Ainsi, si l'évolution avancée dans l'historiographie traditionnelle est bien globalement valable, il est manifeste que de nombreuses subtilités demandent à être prises en compte, sans même rentrer particulièrement dans les détails.

Figure 6. Quelques exemples de tenues courantes

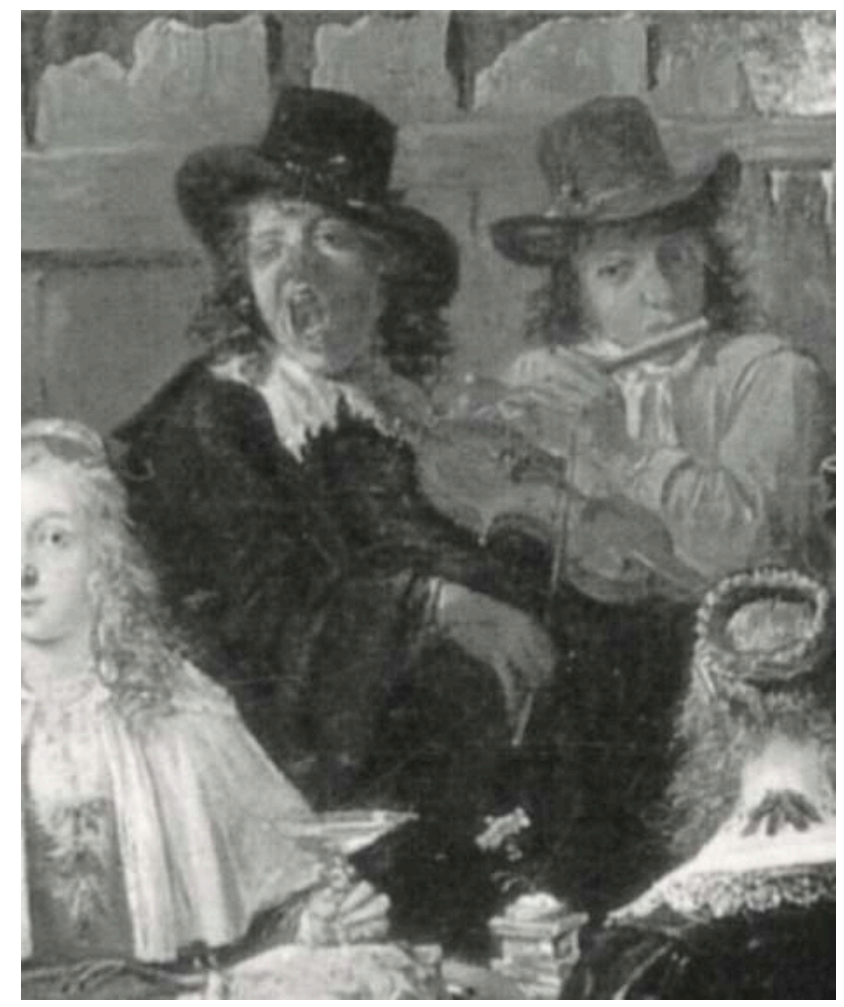

Violon bas, archet tenu à l'italienne.

TENIERS, David le Jeune, Le fils prodigue, 1644, huile sur cuivre, 70 x 89 cm, Paris, Musée du Louvre. Détail. 


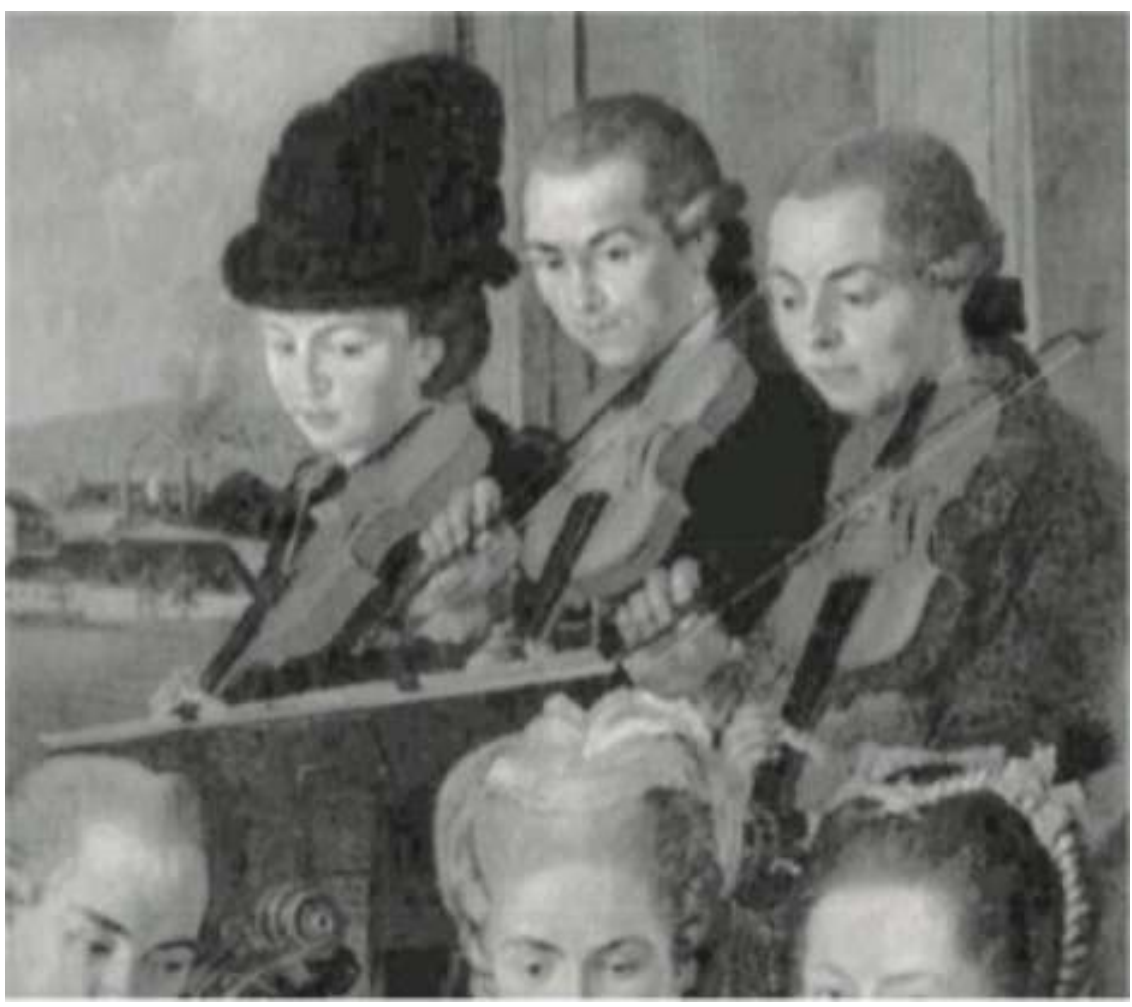

Violon haut, archet tenu à l'italienne.

ZICK, Januarius, La famille Remy, 1776, huile sur toile, 200 × 276 cm, Nuremberg, Germanisches Nationalmuseum. Détail.

\section{Quelques études de cas}

Parallèlement à ces considérations d'ordre général, il peut être intéressant de se pencher sur quelques cas particuliers, moins communs, qui permettent de mettre en avant les particularités méthodologiques de cette recherche. Il est en premier lieu particulièrement intéressant de se focaliser sur le personnage de Nicola Matteis. Celuici, originaire de Naples, vint s'installer à Londres dans les années 1670, pour profiter jusqu'à sa mort, vers 1698 , de son incontestable supériorité technique sur les musiciens anglais et faire une carrière de virtuose. Il n'en existe aucun dessin ou portrait, mais plusieurs descriptions écrites témoignent de la surprise des anglais face à sa technique, puisqu'il aurait porté son violon "contre les côtes courtes" et presque sur sa ceinture $^{12}$. Ce compositeur a fait l'objet du seul disque enregistré à l'heure actuelle en recherchant une tenue historiquement exacte ${ }^{13}$. Or, les termes rencontrés dans les sources ne correspondant absolument pas à ce que l'on connait habituellement, les " côtes courtes » ont été interprétées comme la clavicule et la mention de la ceinture négligée, conduisant finalement l'interprète à opter pour une tenue à l'épaule. Pourtant, cette dernière se voit couramment dans les gravures anglaises de cette époque, et il n'y aurait donc eu aucune raison pour les spectateurs de Matteis de s'étonner... Il suffit en réalité de vérifier dans un ouvrage d'anatomie du XVII ${ }^{e}$ siècle pour comprendre que les «côtes courtes» sont les côtes flottantes, à savoir les trois plus basses de la cage thoracique ${ }^{14}$ : ce violoniste tenait donc bien son instrument quasiment sur le ventre, ce qui concorde également avec les mentions de ceinture et l'étonnement du public. De plus, une telle posture est représentée clairement sur un 
tableau hollandais contemporain de Matteis (sans doute sans rapport avec lui, mais qui prouve qu'elle pouvait exister à l'époque), et l'expérimentation démontre que ses morceaux sont plus simples à jouer ainsi qu'en tenant le violon à l'épaule [fig.7]. Ce premier exemple montre bien l'importance de la méthode historique dans l'approche de ce type de problématique.

Figure 7. Une tenue très basse, comparable à celle de Nicola Matteis

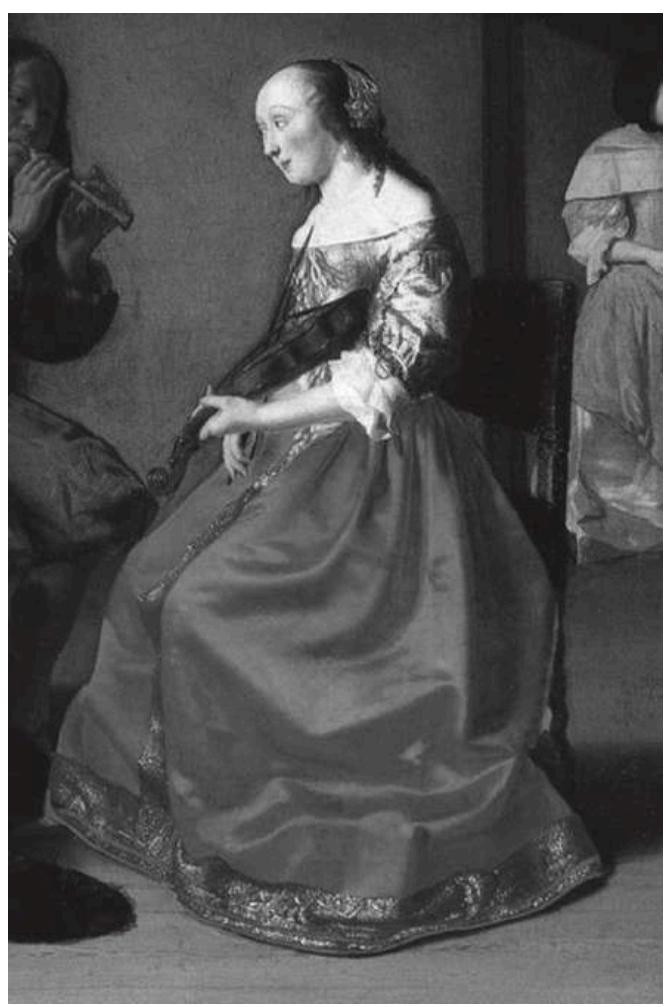

OCHTERVELT, Jacob, Assemblée jouant de musique en intérieur, v.1670, huile sur toile, 59 × 49 cm, Cleveland, Museum of Art. Détail.

A l'inverse, faute de sources textuelles, les trois tenues les moins « classiques » ont été totalement ignorées dans l'historiographie. Celle qui aurait probablement pu être le plus aisément mise en lumière consiste à coincer le violon sous le bras droit, car elle est représentée dans le très célèbre Repas des paysans des frères Le Nain (1642) [fig.6]. Il s'agit de l'une de seules attestations claires de cette technique, avec Le Violoniste de Gerard Ter Borch (v.1665). On pourrait croire, comme dans la majorité des autres tableaux où cette tenue est représentée, que le personnage se prépare ou s'accorde, ce qui est généralement supposé par les analystes. Pourtant, dans ces deux cas, la main gauche est clairement en position de jeu, ce qui exclut la possibilité d'un accord, et l'archet est directement au contact des cordes, position à la fois inutile (car produisant souvent des bruits parasites) et inconfortable si l'on se prépare à jouer. De plus, il est possible de trouver quelques attestations de l'existence de cette technique dans l'ethnographie, notamment à travers quelques photographies de violoneux prises lors de mariages percherons au début du $\mathrm{XX}^{\mathrm{e}}$ siècle. L'expérimentation ayant enfin démontré qu'il est possible de jouer dans cette position, quoique le son produit ne soit ni très propre ni très puissant, nous en déduisons, cette fois principalement grâce à 
l'iconographie, que cette tenue, bien qu'exceptionnelle, a certainement dû être utilisée ponctuellement.

Figure 8. Exemples de tenue sous le bras droit

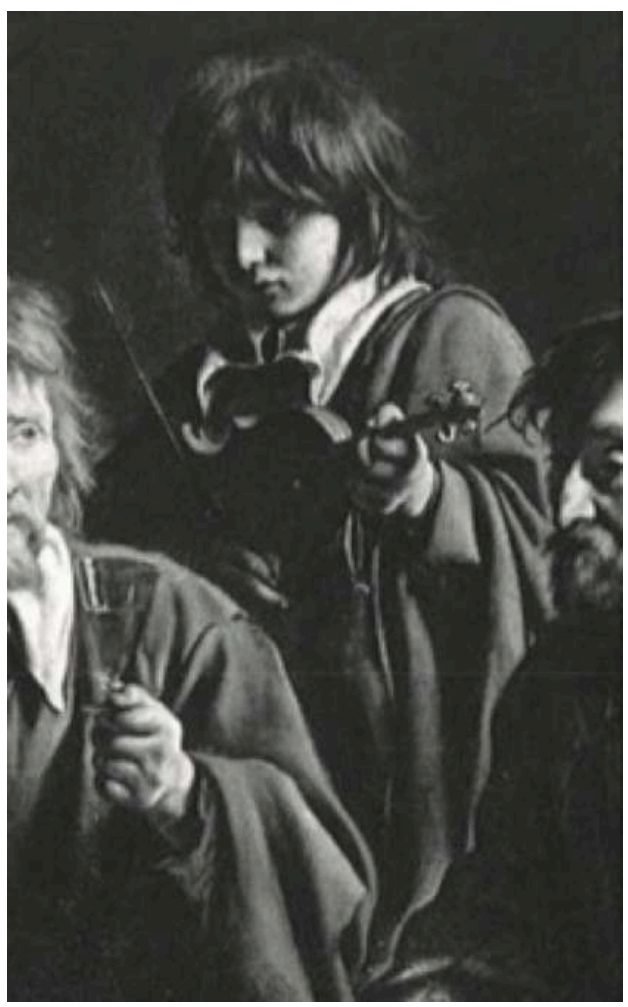

LE NAIN, Le repas des paysans, 1642, huile sur toile, 97 × 122, Paris, Musée du Louvre. Détail.

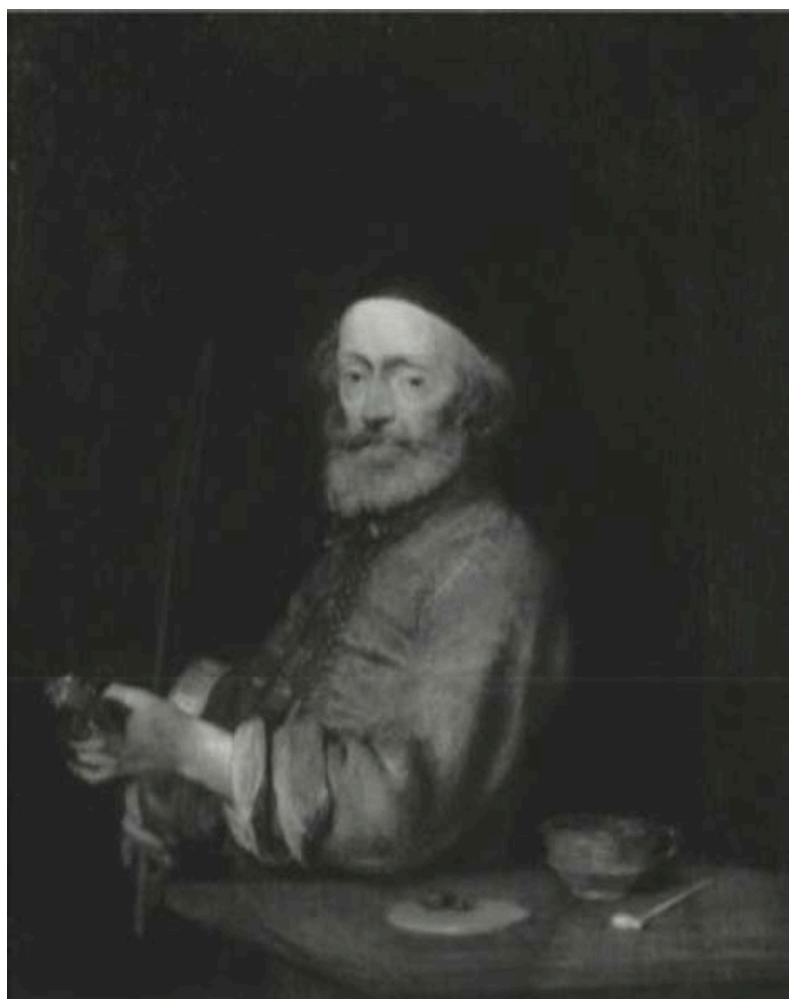

TERBORCH, Gerard, Le violoniste, v.1665, huile sur bois, 29 × 24 cm, Saint-Pétersbourg, Ermitage. 


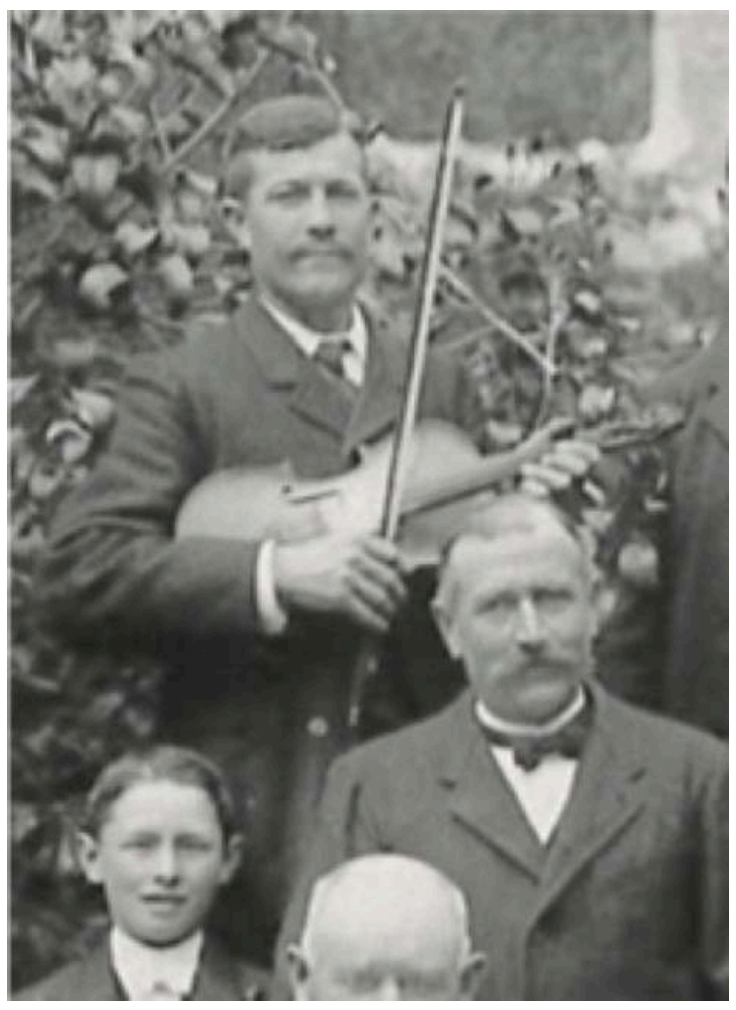

Violoneux lors d'un mariage au Pin-la-Garenne (Orne), 1910. Détail. Courtoisie de l'Écomusée du Perche.

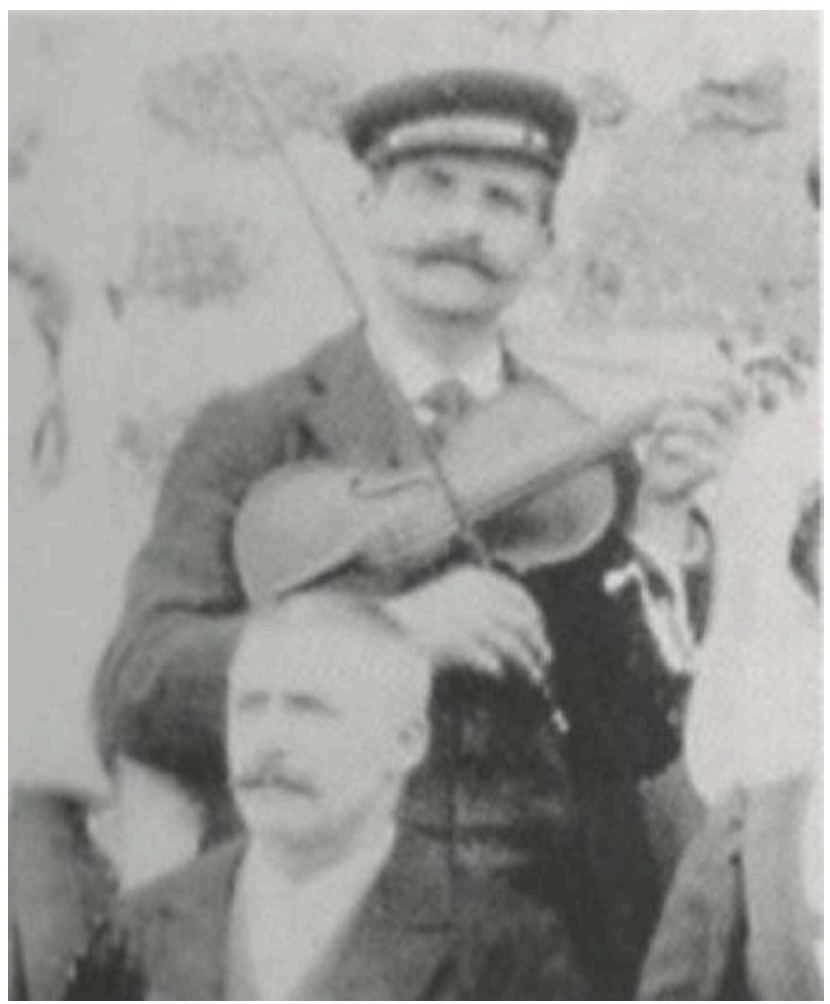

Violoneux lors d'un mariage dans le Perche, début du XXe siècle. Détail. L

EROUX, Jean-François, Trésor de la musique traditionnelle dans le Perche, Rémalard, Fédération des Amis du Perche, 2005, 280 p. 
Dans d'autres cas, la solution peut provenir d'un recours à l'ethnographie, notamment pour une tenue contre l'épaule droite. Cette position, dont il n'est jamais question dans les sources textuelles, est pourtant représentée à quelques occasions dans l'iconographie, et en particulier en Italie au XVI ${ }^{\mathrm{e}}$ siècle, en Suisse au Moyen-Âge et en France au XVII ${ }^{\mathrm{e}}$ siècle (une occurrence). La plupart de ces représentations prennent place dans des compositions religieuses et ne sont pas toujours réalistes, mais quelques-unes semblent tout de même assez crédibles et nous obligent à poser la question de l'existence de ce geste technique. Or, il se trouve que cette position est attestée chez certains musiciens tziganes actuels, ce qui, à défaut de prouver son utilisation à l'époque baroque, permet de supposer que les représentations ne sont pas forcément erronées [fig.7].

Figure 9. Tenue contre l'épaule droite

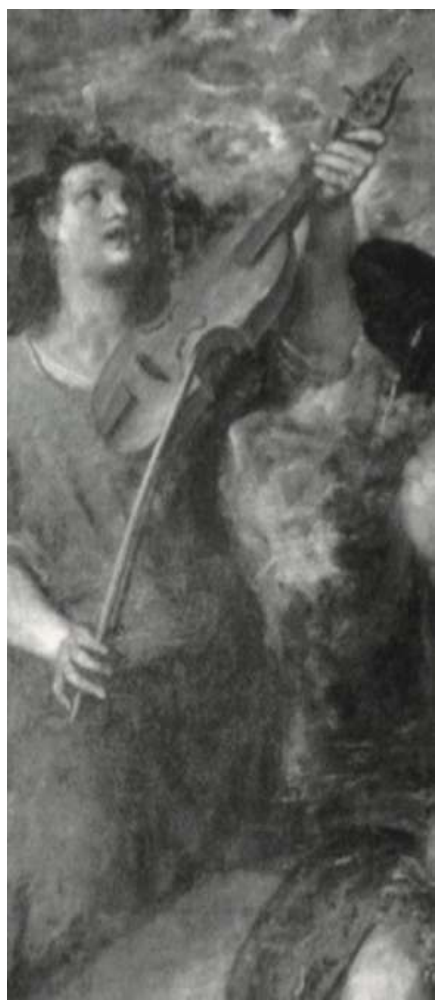

TIZIANO, Vecellio, Le supplice de Marsyas, 1576, huile sur toile, 212 × 207 cm, Kromeriz, State Museum. Détail. 


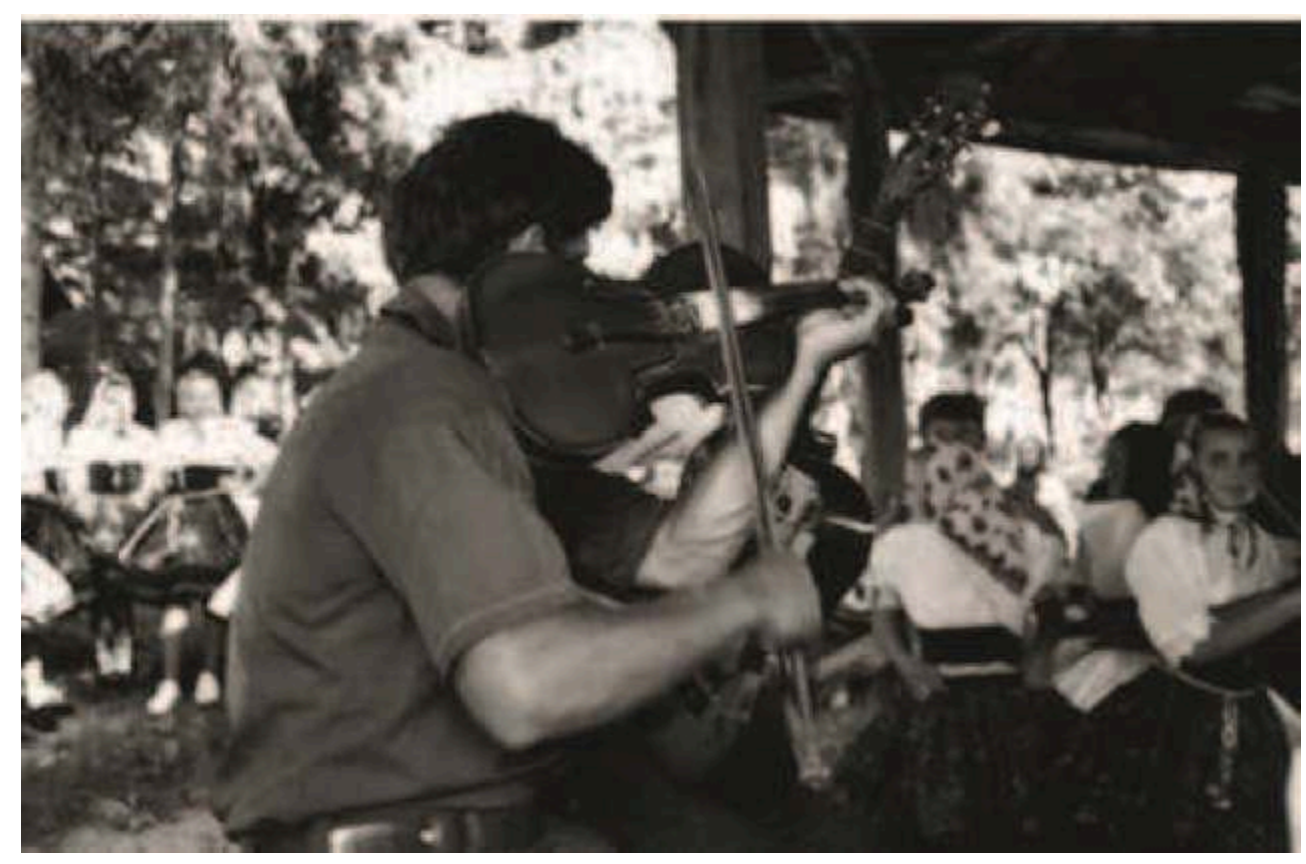

Violoneux lors d'un bal à Racșa (Roumanie), 1998.

Photo Jacques Bouët, in BOUET, Jacques, « Déterminé ma non troppo. Une forme signifiante en pleine efflorescence au Pays de l'Oach (Roumanie) revisité dans le sillage de Béla Bartók », Cahiers de musiques traditionnelles, 17, 2004, p.161-182.

Le recours à l'ethnologie peut cependant poser certains problèmes qu'un dernier exemple peut nous aider à cerner, celui-ci faisant plutôt intervenir l'expérimentation comme méthode principale. Il s'agit du fait de tenir le violon verticalement, en général posé sur une table. Si quelques attestations existent dans un cadre religieux en Allemagne au début du XVI ${ }^{e}$ siècle ou en Italie au début du XVII ${ }^{e}$, une peinture de Pieter De Hooch, représentant une scène réaliste en Hollande en 1677, amène là encore à interroger la validité ou non de cette iconographie [fig.8]. Un regard vers l'ethnographie nous montre que les musiciens traditionnels arabes emploient effectivement le violon posé parfaitement vertical sur les genoux, en le faisant pivoter selon la corde à jouer. Or, il apparaît lors de l'expérimentation que reproduire cette tenue sur une table, en faisant reposer le violon sur le bouton, est extrêmement malaisé pour la musique occidentale, l'instrument étant difficilement maintenable en position : le parallèle ethnographique n'est donc probablement pas pertinent. Pourtant, un examen plus attentif des représentations picturales montre que le violon n'y est jamais représenté strictement vertical mais toujours légèrement penché. C'est alors qu'une nouvelle expérimentation démontre qu'en stabilisant le violon sur les éclisses au lieu de le faire reposer sur le bouton, les problèmes évoqués précédemment disparaissent. Ainsi, non seulement l'expérimentation démontre ici la faisabilité technique de cette position, mais met également en évidence le fait que les peintres ont représenté l'instrument dans la seule position techniquement viable pour une tenue verticale, ce qui appuie nettement l'hypothèse qu'ils auraient effectivement observé des musiciens tenir leur violon de cette manière. 
Figure 10. Tenue verticale

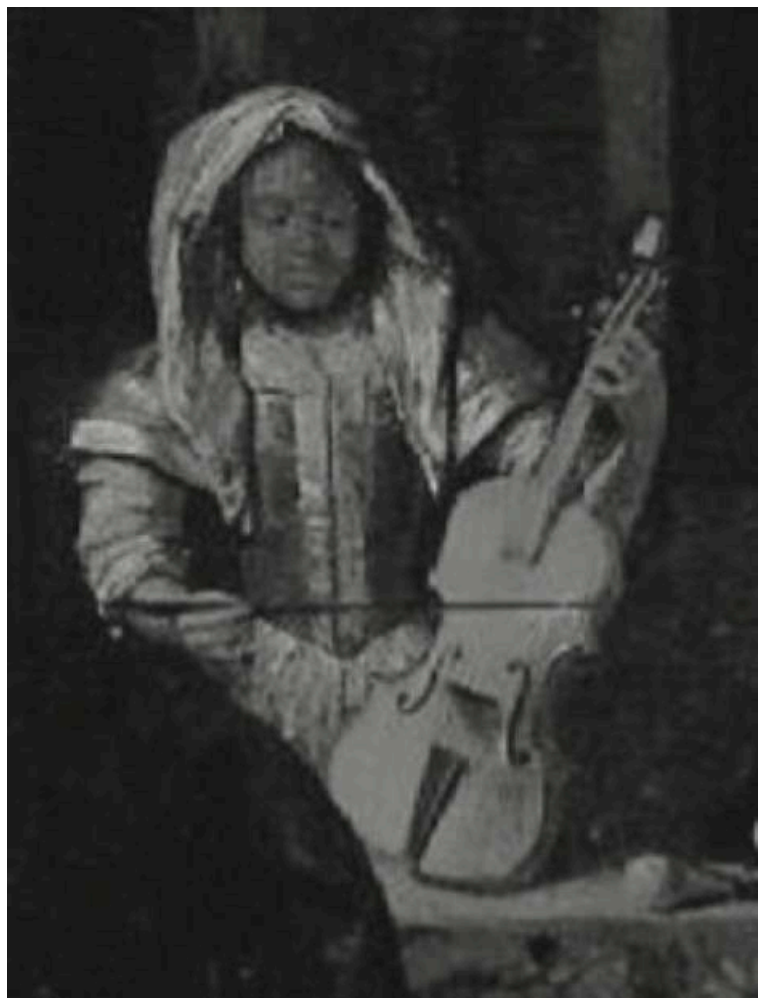

DE HOOCH, Pieter, Réunion musicale dans une cour, 1677, huile sur toile, 83,5 × 68,5 cm, Londres, National Gallery. Détail.

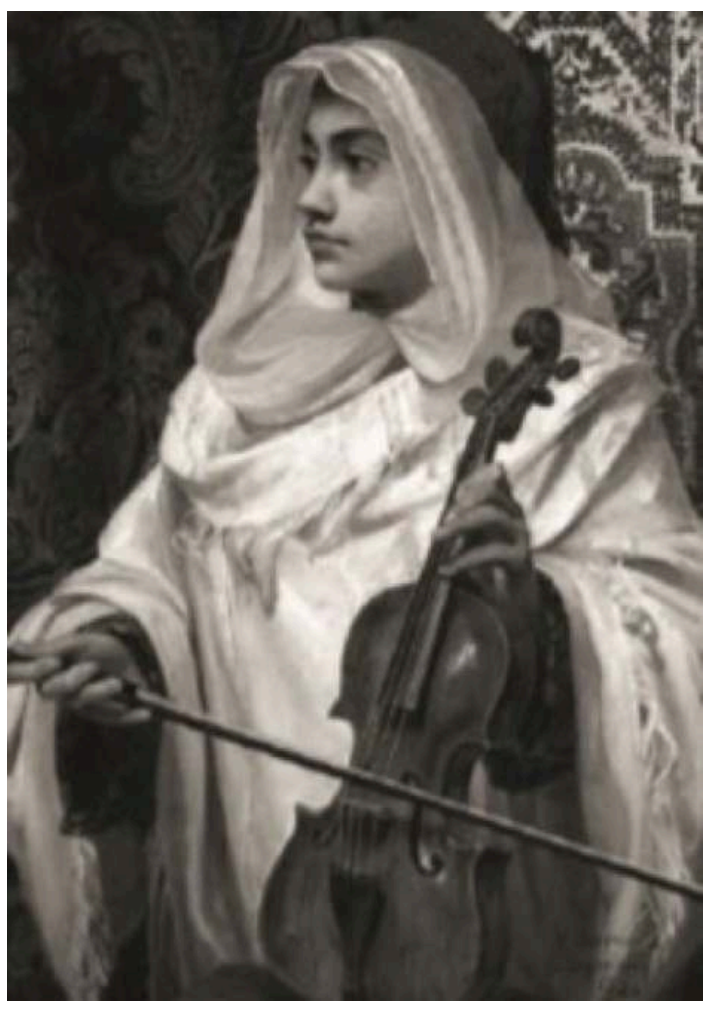

AMERICO, Pedro, Le joueur de rebec, 1884, huile sur toile, 87,6 × 60 cm, Rio de Janeiro, Museu Nacional de Belas Artes. 


\section{Implications sur la recherche et conclusion}

\section{Poursuite de la réflexion}

19 Si nous avons pu, grâce à l'abondance des sources analysées de manière systématique, mettre en évidence un certain nombre de dynamiques et de cas particuliers dans l'évolution de la tenue du violon à l'époque baroque, il n'en reste pas moins difficile de franchir cette étape et de tenter d'avancer des hypothèses quant au pourquoi de ces observations. Il est évidemment important de se départir de la vue téléologique héritée des théoriciens du XIX siècle, selon laquelle la technique du violon aurait simplement suivi une évolution à sens unique, d'une pratique populaire vers une configuration savante, au même titre que la musique. En effet, outre que ce type de réflexion ne peut jamais être considérée comme historiquement valide, il est clair au regard des observations précédemment formulées que la tenue du violon n'a pas évolué à sens unique, ne serait-ce que parce que la tenue moderne semble beaucoup plus proche de celle de la Renaissance que des différentes formes observées au XVII ${ }^{\mathrm{e}}$ siècle.

Il est cependant notable que la tenue utilisée est au moins en partie liée à la musique elle-même. En effet, certaines difficultés techniques requises dans des morceaux de la fin de l'époque baroque (chez l'italien Locatelli par exemple) ne sont réalisables qu'avec une tenue quasiment moderne, qui permet de tenir le violon avec le menton pour libérer la main gauche. A l'inverse, les partitions anglaises des XVII et $\mathrm{XVIII}^{\mathrm{e}}$ siècles ne présentent pas du tout le même type de technicité, et il est possible de les jouer avec toutes les tenues énumérées précédemment: dans ce cas, le choix de l'une ou l'autre est plutôt lié à la recherche de confort, ou à un effet de mode. Il a notamment été avancé que le fait que la tenue ait tendance à migrer de la poitrine vers le cou dans les représentations de la bonne société à partir de la fin du XVII siècle, soit lié à la volonté de s'approcher de la tenue des solistes pour des raisons de représentation sociale ; mais dans ce cas, on peut se demander dans quelle mesure la pratique concrète a évolué réellement, et dans quelle autre mesure seule la représentation picturale aurait pu changer.

Enfin, il faut souligner l'importance de prendre en compte la matérialité de l'instrument lors d'une telle étude, notamment en ce qui concerne l'archet, élément indissociable du violon. En effet, le violon lui-même a assez peu changé entre le XVI ${ }^{e}$ et le XVIII ${ }^{e}$ siècle, du moins en ce qui concerne les éléments susceptibles d'influer sur la tenue. Par contre, l'archet a été profondément modifié dans le même laps de temps, passant d'un arc court et à forte courbure convexe à un archet long, non courbé voire quelque peu concave. Bien que la brièveté du propos ne nous permette pas ici de traiter cet aspect en détail, il convient donc de garder à l'esprit que les modifications quant à la tenue de l'archet peuvent être très fortement liées aux évolutions de celui-ci: la longueur de mèche utilisable serait ainsi la même en tenant au milieu un archet long ou à l'extrémité un archet court, alors que le fait de tenir de la même manière des archets de tailles différentes peut modifier grandement les possibilités de jeu. Afin de tenir compte au mieux de ces modifications, il serait donc essentiel de mener une étude archéologique de l'archet (à partir des représentations puisque les instruments d'époque encore en l'état se comptent sur les doigts d'une main), avant de pouvoir effectuer une analyse détaillée des évolutions de sa tenue. 


\section{Interdisciplinarité et « histoire appliquée »}

Le terme d'« histoire appliquée ", apparu assez récemment pour désigner le fait de tester concrètement les éléments historiques auparavant traités de manière uniquement abstraite, pourrait s'appliquer à cette analyse de la tenue du violon. En effet, il semble indispensable de tester les résultats obtenus en situation réelle de jeu, et il paraît également impensable de traiter de ce sujet sans en donner une petite démonstration. Cependant, nous pensons que cette recherche doit s'inscrire dans un cadre plus large : en effet, l'instrument est également utilisé pour une expérimentation en règle, assez proche de ce qui peut se pratiquer en archéologie des techniques (bien que sans dimension archéométrique), et seul le concert "historiquement informé " peut être considéré comme de l'histoire appliquée ou de la reconstitution. Mais il faut aussi souligner l'importance de la pluridisciplinarité dans cette étude: celle-ci regroupe en effet un travail d'historien, ainsi que de "technicien» (puisqu'il faut impérativement maîtriser l'instrument pour mener celle-ci à bien, ou du moins dans de bonnes conditions), mais implique également un détour par l'histoire de l'art, l'ethnographie, ou même l'archéologie. On pourrait ainsi même poser la question de la pertinence d'appeler cette recherche de l' "histoire» tant ces différentes sources semblent se mêler sans que l'une ne soit plus importante que les autres : peut-être ce type de sujet de recherche, particulièrement propre à l'histoire des techniques, annonce-t-il un renouvellement méthodologique et épistémologique de la discipline historique, qui sans la remettre en cause en elle-même viendrait l'ouvrir de manière novatrice vers les autres champs des sciences humaines, voire des sciences « dures".

Nous nous devons également de souligner l'importance des nouvelles technologies dans cette recherche, ceci expliquant en grande partie pourquoi de telles analyses n'ont pas pu être menées avant les années 2000. En effet, les sources textuelles et iconographiques utilisées ici sont très peu diffusées dans le premier cas, extrêmement nombreuses et diverses dans le second, et dispersées dans le monde entier dans les deux. Nous n'avons donc pu les compiler et les analyser de manière massive que par le biais de l'Internet collaboratif. En effet, la plupart des traités utilisés ont été numérisés et placés en ligne par des passionnés totalement bénévoles, sur un site dédié à la diffusion de partitions libres de droit (particulièrement utile en musique baroque où les éditions sont assez rares) ${ }^{15}$, et l'iconographie a été intégralement recensée en parcourant en intégralité deux bases de données non académiques répertoriant des œuvres picturales conservées dans des musées du monde entier ${ }^{16}$. Faute de disposer d'équivalent académique, du moins en France (des bases de données allemandes et américaines nous ont par contre été utiles), ce type de recherche nécessitant l'analyse de très nombreuses sources particulièrement dispersées ne peut donc être menée à terme dans des délais raisonnables que par l'intermédiaire du web 2.0, et de toute manière, avec ou sans support académique, par le truchement des technologies informatiques. 


\section{NOTES}

1. DUBOURG GLATIGNY Pascal, VÉRIN Hélène, Réduire en art. La technologie de la Renaissance aux Lumières, Paris, Maison des Sciences de l'Homme, 2008.

2. BOYDEN David, The history of violin playing from its origins to 1761 and its relationship to the violin and violin music, Oxford, Oxford University Press, 1990 (1ère éd. 1965).

3. BURGOS Jean-Paul, « Des racleurs de contredanse ». Tenue et jeu du violon, aux XVIIe et XVIIIe siècles », Flûte à bec \& instruments anciens, 16, octobre 1985, p.8-15.

4. MOENS-HAENEN Greta, Deutsche Violintechnik im 17. Jahrhundert. Ein Handbuch zur Aufführungspraxis, Graz, Akademische Druck- u. Verlagsanstalt, 2006.

5. CIZERON Janine, « Les méthodes de violon françaises aux XVIIe et XVIIIe siècles », in PENESCO, Anne (dir.), Itinéraires de la musique française. Théorie, pédagogie et création, Lyon, PUL, 1996, p.87-103.

6. STOWELL Robin, The Early Violin and Viola. A Practical Guide, Cambridge, Cambridge University Press, 2001, p.1-9.

7. GEMINIANI Francesco, The Art of Playing on the VIOLIN. Containing All the Rules necessary to attain to a Perfection on that Instrument, with great variety of Compositions, which will also be very useful to those who study the Violoncello, Harpsichord \&c, London, John Johnson, 1751.

8. MOZART Léopold, Méthode raisonnée Pour apprendre à Jouer du Violon, Paris, Le Menu, 1770.

9. CORRETTE Michel, L'École d'Orphée. Méthode Pour Apprendre facilement à Joüer du Violon Dans le goût François et Italien; Avec des Principes de Musique Et beaucoup de Leçons à I, et II Violons. Ouvrage utile aux commençants et a ceux qui veulent parvenir à l'exécution des Sonates, Concerto, Pièces par accords Et Pièces à cordes Ravallées, Paris, L'Auteur, Boivin et Le Clerc, 1738. 10. PRINNER Johann, Musicalischer Schlissl, Salzburg, 1677, s.p.

11. MOZART Léopold, op. cit.

12. NORTH Roger, The Musicall Gramarian, 1728, s.p.

13. Matteis. False consonances of melancholy, Gli Incogniti (violon Amandine Beyer), Paris, Zigzag Territoires, 2009, 40 pistes.

14. VAN DEN SPIEGEL Adrian, The Workes of that Famous Chirurgion Ambrose Parey, Londres, Richard Cotes et Willi Dugard, 1649, p.108

15. L'International Music Score Library Project : http://imslp.org.

16. La Web Gallery of Art : http://www.wga.hu/. La bibliothèque allemande en ligne Zeno : http:// www.zeno.org/.

\section{RÉSUMÉS}

La question de la tenue du violon pendant ses deux premiers siècles d'existence n'a jamais été traitée exhaustivement dans l'historiographie récente. Il s'agit pourtant d'une thématique particulièrement intéressante dans un domaine où l'application des études historiques, à travers les concerts de musique ancienne, constitue la motivation principale des recherches. Les sources disponibles sont particulièrement variées et couvrent une grande partie de l'éventail potentiel des sources en histoire des techniques : quelques mentions écrites, beaucoup d'iconographie, un emploi occasionnel de l'ethnographie et de l'archéologie, et enfin un recours régulier à 
l'expérimentation. Nous proposons ici une analyse globale de l'évolution dans le temps et l'espace de la tenue du violon et de l'archet entre le XVIe et le XVIIIe siècle, qui conduit à préciser de nombreux points restés succincts dans la bibliographie. D'autre part, afin d'expliciter les mécanismes méthodologiques de cette recherche, nous nous focalisons également sur quatre cas particuliers, avec des tenues très éloignées des habitudes actuelles de la musique classique, et jamais traitées auparavant. Cette étude, encore en cours, met ainsi en évidence l'intérêt, voire ici la nécessité, d'une approche pluridisciplinaire de ce type de problématique, et dans notre cas la nécessité pour le chercheur de maîtriser lui-même techniquement son objet d'étude, puisque l'expérimentation est une composante incontournable de notre méthodologie. Enfin, une telle étude n'a pas été possible jusqu'à récemment, notamment pour la partie iconographie, car celleci nécessite le traitement d'un grand nombre de documents très dispersés, qui ne sont réunis que dans des bases de données collaboratives en ligne : ce constat appelle une réflexion sur l'importance de ces outils, impliquant la participation massive de passionnés non professionnels, dans la recherche actuelle.

The question of the holding of the violin during the first two centuries of its existence has yet to be exhaustively studied within recent historiography. Despite this, it is a topic of particular interest within a field of study for which historical exploration, through concerts of ancient music, is the main motivation for research. Available sources are particularly varied and include a large part of the various kinds used in the history of technology, such as written records, copious illustrations, the occasional application of ethnography and archeology, and, finally, the conducting of trials. We here propose an analysis of the evolution in space and time of the techniques of holding the violin and bow between the sixteenth and eighteenth centuries, which permits the clarification of a number of points not yet fully explored within the available literature. On the other hand, in order to clarify the methodological mechanisms of this research, we shall also focus on four specific cases using techniques far removed from current trends in classical music and that have been heretofore undealt with. This study, still in progress, highlights the interest in, or even the need of, a multidisciplinary approach to this type of problem, and, in this specific case, the need for the researcher to technically master the object of study, as experimentation is an essential component of our methodology. Finally, such a study, including the section concerning iconography, has not been possible until recently, due to the necessity of processing a large number of documents widely dispersed, which have only been fully assembled in collaborative databases online: This observation calls for a reflection on the importance of such tools that involve the massive participation of passionate nonprofessionals within current research.

\section{INDEX}

Mots-clés : histoire des techniques, énoncé technique, époque moderne, violon, geste, expérimentation, iconographie, histoire appliquée

Keywords : history of technology, technical writing, modern period, violin, gesture, experimentation, iconography, applied history

\section{AUTEUR}

\section{CYRIL LACHEZE}

Masterant en Histoire des Techniques, Masterant en Archéologie, Université Paris I PanthéonSorbonne 